\title{
Three-dimensional structure of Bax-mediated pores in membrane bilayers
}

\author{
X-P Xu', D Zhai ${ }^{2}$, E Kim ${ }^{1,3}$, M Swift ${ }^{1}$, JC Reed ${ }^{2}$, N Volkmann ${ }^{*, 1}$ and D Hanein ${ }^{1}$
}

B-cell lymphoma 2 (Bcl-2)-associated $\mathrm{X}$ protein (Bax) is a member of the $\mathrm{Bcl}-2$ protein family having a pivotal role in triggering cell commitment to apoptosis. Bax is latent and monomeric in the cytosol but transforms into its lethal, mitochondria-embedded oligomeric form in response to cell stress, leading to the release of apoptogenic factors such as cytochrome $\mathrm{C}$. Here, we dissected the structural correlates of Bax membrane insertion while oligomerization is halted. This strategy was enabled through the use of nanometer-scale phospholipid bilayer islands (nanodiscs) the size of which restricts the reconstituted system to single Bax-molecule activity. Using this minimal reconstituted system, we captured structural correlates that precede Bax homo-oligomerization elucidating previously inaccessible steps of the core molecular mechanism by which Bcl-2 family proteins regulate membrane permeabilization. We observe that, in the presence of BH3 interacting domain death agonist (Bid) BH3 peptide, Bax monomers induce the formation of $\sim 3.5-\mathrm{nm}$ diameter pores and significantly distort the phospholipid bilayer. These pores are compatible with promoting release of ions as well as proteinaceous components, suggesting that membrane-integrated Bax monomers in the presence of Bid BH3 peptides are key functional units for the activation of the cell demolition machinery.

Cell Death and Disease (2013) 4, e683; doi:10.1038/cddis.2013.210; published online 20 June 2013

Subject Category: Experimental Medicine

B-cell lymphoma 2 (Bcl-2) family proteins are central regulators of cell death, ${ }^{1-3}$ sharing regions with similar amino-acid sequence, referred to as Bcl-2 homology motifs $\mathrm{BH} 1,2,3$ or 4 . The superfamily can be divided into three classes, with one class promoting cell death, the other promoting cell survival and the third class (the BH3-only proteins) serving as upstream cell-stress sensors or regulators. In general, the pro-apoptotic proteins contain the $\mathrm{BH}$ domains 1-3 whereas the anti-apoptotic class contains $\mathrm{BH}$ domains 1-4. The spatiotemporal interactions between members of the three classes in the vicinity of the mitochondria determine cell fate. Insights into the biochemical, cellular and physiological roles of the $\mathrm{Bcl}-2$ family members have shown that the $\mathrm{BH} 3$ class transmits pro-death signals to the apoptotic machinery located at the mitochondrial membranes. This signal, once delivered to pro-apoptotic proteins such as $\mathrm{Bcl}-2$-associated $\mathrm{X}$ protein (Bax), triggers cell commitment to apoptosis via the mitochondrial outer membrane permeabilization (MOMP) mechanism. The latter involves the release of several mitochondrial proteins into the cytoplasm, including cytochrome $\mathrm{C}$, which activates caspases that in turn initiate the apoptotic cell death program. The anti-apoptotic protein B-cell lymphoma-extra large (Bcl-xL) inhibits Bax-mediated MOMP.

Much of the current thoughts on the core molecular mechanism by which $\mathrm{Bcl}-2$ family proteins, more specifically
Bax and $\mathrm{Bcl}-\mathrm{xL}$, regulate membrane permeabilization are inferred from a combination of biophysics, cell-free systems and molecular biology approaches. Although it is generally agreed that BH3-only proteins promote Bax-mediated membrane permeabilization and their synergistic effect suggests a role in direct Bax activation, the underlying mechanism is under intense study, and is often the subject of considerable debate. ${ }^{2,4}$

In vitro studies have shown that membrane permeabilization occurs through an ordered series of steps. Inactive Bax resides mainly in the cytoplasm. The transition of Bax into its pro-apoptotic active form includes a reversible membranebinding step that is distinct from membrane integration. ${ }^{5}$ The integration of Bax into the membrane is the rate-limiting step of the process ${ }^{6}$ and Bax undergoes major conformational changes during this stage. ${ }^{6,7}$ Various studies have suggested that the membrane acts as part of a combinatorial input framework with activating $\mathrm{BH}$-only proteins to induce Bax unfolding and insertion into the membrane, thus promoting Bax activation. ${ }^{8,9}$ Additional upstream effectors further support Bax insertion. ${ }^{10} \mathrm{Bax}$ is capable of permeabilizing liposome vesicles when stimulated with a $\mathrm{BH} 3$ peptide from $\mathrm{BH} 3-$ only proteins such as $\mathrm{BH} 3$ interacting domain death agonist (Bid). ${ }^{9,11}$

Major conformational rearrangements and extensive homooligomerization of Bax has been observed in the presence of

\footnotetext{
${ }^{1}$ Bioinformatics and Systems Biology Program, Sanford-Burnham Medical Research Institute, La Jolla, CA 92037, USA and ${ }^{2}$ Apoptosis and Cell Death Research Program, Sanford-Burnham Medical Research Institute, La Jolla, CA 92037, USA

*Corresponding author: N Volkmann, Bioinformatics and Systems Biology Program, Sanford-Burnham Medical Research Institute, 10901 N Torrey Pines Road, La Jolla, CA 92037, USA. Tel: 858-646-3100; Fax: 858-646-3195; E-mail: niels@ burnham.org

${ }^{3}$ Present address: MSM Protein Technologies, Inc., Medford, MA 02155, USA.

Keywords: Bax; apoptosis; electron cryo-microscopy; 3D image reconstruction; nanodiscs

Abbreviations: Bax, Bcl-2-associated X protein; Bcl-2, B-cell lymphoma 2; Bcl-xL, B-cell lymphoma-extra large; Bcl-xL $\Delta T \mathrm{TM}, \mathrm{Bcl}-\mathrm{xL}$ with transmembrane domain removed; $\mathrm{BH} 3$, Bcl-2 homology motif 3; Bid, BH3 interacting domain death agonist; cryo-EM, electron cryo-microscopy; DPPC, dipalmitoylphosphatidylcholine; FITC, fluorescein isothiocyanate; MOMP, mitochondrial outer membrane permeabilization; MSP1, membrane scaffold protein 1; tBid, truncated Bid; 3D, three-dimensional Received 09.5.13; accepted 14.5.13; Edited by G Melino
} 
detergent, ${ }^{12}$ leading, by inference, to a model where integration of Bax into the membrane is associated with homooligomerization followed by membrane permeabilization. So far, the exact temporal sequence of events remains elusive. As part of this process, Bax oligomerization is thought to occur sometime after membrane association, leading to large, irregular mixed protein-lipidic pores such as those observed by electron cryo-microscopy (cryo-EM) of large unilamellar vesicles. ${ }^{13}$ However, the detailed spatiotemporal, molecular mechanism of Bax-mediated pore formation remains to be further defined and the explicit structure of membraneembedded Bax and how it gives rise to a functional pore remains an active area of investigation.

Here, we used high-resolution cryo-EM to determine the three-dimensional (3D) structures of nanometer-scale, protein-supported monodisperse phospholipid particles (nanodiscs), ${ }^{14}$ in the presence and absence of full-length human Bax, Bid BH3 peptide and Bcl-xL. The small size of nanodiscs ( 12-nm diameter) and their narrow size distribution (2-3\% deviation in number of lipid molecules) ensures that, under well-determined biochemical conditions, only single Bax molecules are incorporated into nanodiscs, thus preventing the possibility that interactions between Bax molecules (such as dimerization/oligomerization) have an effect on the imaged results of our studies. The restriction of the reconstituted system to single Bax molecule activity allows us capturing a snapshot of MOMP that precedes Bax homo-oligomerization. We observe that nanodiscs form elliptical lipid bilayers islands delimited by a protein belt. In the presence of Bid $\mathrm{BH} 3$ peptide, incorporation of Bax-monomers significantly distorts the nanodisc bilayer and produces a pronounced pore in the lipid bilayer. Bcl-xL prevents Bax incorporation. Furthermore, the observed Bax-induced pores, with an average diameter of $\sim 3.5 \mathrm{~nm}$, are compatible with release of ions and protein components including cytochrome $\mathrm{C}$. Thus, here we provide direct evidence that Bax monomers, once activated by Bid BH3 peptide and incorporated in a lipid bilayer, induce the formation of pores and permeabilize the membrane bilayer.

\section{Results}

Minimal reconstituted systems that included lipid membrane (liposomes), activator protein (truncated Bid, tBid) or Bid BH3 peptides, as well as pro-survival protein ( $\mathrm{Bcl}-\mathrm{xL}$ ) served as powerful systems to study Bax activation and membrane permeabilization. , $8,9,13,15-17$ These studies allowed unraveling some of the critical steps involved in the mechanism of Bax-mediated MOMP and provided the first observations for the existence of Bax-induced pores.

Here we used a platform technology that allows dissection of the structural correlates of Bax membrane insertion while oligomerization is halted. To achieve this feat, we used nanometer-scale, protein-supported phospholipid particles (nanodiscs). ${ }^{14}$ These nanodiscs are of defined size small enough to prevent incorporation of Bax oligomers. As importantly these bilayers also recapitulate the properties of biological membranes more closely than liposomes. ${ }^{18}$ The biophysical properties of nanodiscs have been extensively characterized and it was shown that they have a very narrow size distribution $( \pm 2$ to $3 \%$ variation in phospholipid content), ${ }^{14}$ making them ideal candidates for structure determination by cryo-EM and single particle analysis. As Bid $\mathrm{BH} 3$ peptides and phospholipid bilayer are reported to activate Bax at a membrane interface, ${ }^{9}$ the system used in this study represents a minimal system for reconstituting activation and membrane insertion of soluble full-length human Bax monomers.

Bax monomers integrate into nanodiscs in the presence of Bid $\mathrm{BH} 3$ peptide but not when $\mathrm{Bcl}-\mathrm{xL}$ is present. To ensure incorporation of Bax monomers only, we first established a biochemical assay to assess and optimize integration of soluble Bax into nanodiscs. We took advantage of the fact that, consistent with their narrow size distribution, nanodiscs migrate into native gels as a prominent band ( $\sim 220 \mathrm{kDa}$ marker, Figure 1a). Aided by Coomassie staining, western blotting and labeling with fluorescent markers, we were then able to follow colocalization of the respective proteins with the characteristic nanodisc band. If nanodiscs are mixed with fluorescently labeled Bax, no shifts or extra bands occur and the absence of fluorescence showed that Bax by itself does not associate with nanodiscs (Figure 1a). However, when fluorescently labeled Bax and nanodiscs were incubated together with Bid $\mathrm{BH} 3$ peptide, then an additional clear, single band appeared by Coomassie staining at $\sim 250 \mathrm{kDa}$ marker (Figure 1a, left), above the bare nanodisc band. The corresponding fluorescence signal shows a clear and sharp band at the position of the additional Coomassie band, indicating that it represents nanodiscs with incorporated Bax molecules (Figure 1a, right). These findings were fully confirmed using unlabeled Bax and immunoblotting (Figure 1c). Bax incorporation into nanodiscs in the presence of Bid $\mathrm{BH} 3$ peptide was observed to occur at the full range of $\mathrm{pH} 4.0-8.0$, with the most efficient incorporation occurring at $\mathrm{pH} 4$ as judged by the shift in intensities to the stained band (Supplementary Figure S1B). The existence of two bands, the bare nanodisc and Bax-nanodisc assembly bands, occur at substoichiometric Bax concentration and persist at increasing Bax concentrations.

In difference to Bax, fluorescent labeling of Bid $\mathrm{BH} 3$ peptide indicates that the peptide associates with nanodiscs without other factors (Figure 1b). Consistent with its low molecular weight Bid $\mathrm{BH} 3$ peptide association does not significantly shift the nanodisc band position. The Bid $\mathrm{BH} 3$ peptide fluorescence signal was also detected in the additional, second band, which forms when Bax is added to the reconstituted system (Figure 1b, right). Supporting the specificity of the reconstituted system, addition of Bid $\mathrm{BH} 3$ peptide carrying a single point mutation ( $\mathrm{G} / \mathrm{A}$ ) that abolishes Bax binding of Bid in vivo ${ }^{8}$ did not support Bax integration into the lipid bilayer (Figure 1c). Furthermore, the anti-apoptotic protein Bcl-xL abolished Bax integration in the presence of Bid $\mathrm{BH} 3$ peptide (Figure 1d). Altogether, these results indicate that a specific complex between Bax monomers, Bid $\mathrm{BH} 3$ peptide and phospholipids is formed. Bcl-xL inhibits the formation of this assembly.

Nanodiscs form elliptical lipid bilayers delimited by a protein belt. Next, we used a combination of cryo-EM imaging and several image analysis approaches to derive 3D 
a
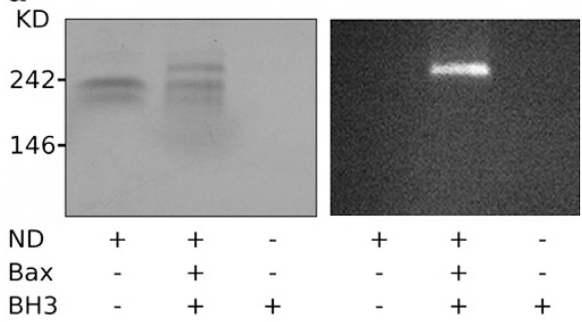

c

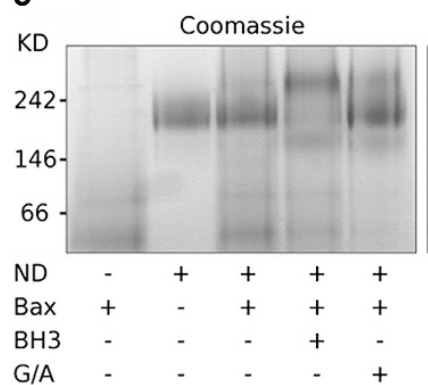

b

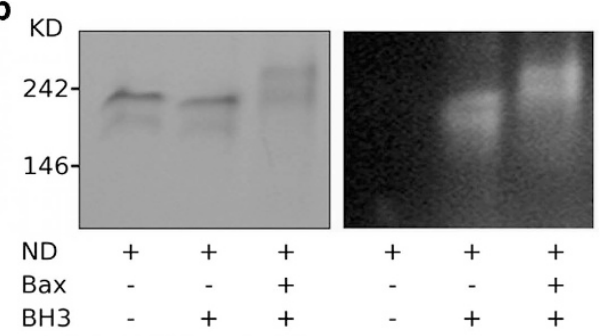

d
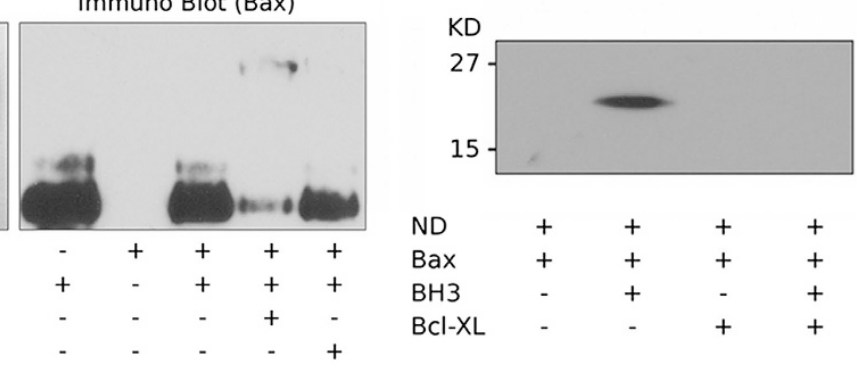

Figure 1 Bax inserts into lipid nanodiscs in the presence of Bid BH3 peptides. (a) Purified recombinant fluorescein-maleimide-labeled Bax, Bid peptides and nanodiscs were analyzed by native gel followed by Coomassie Blue staining and UV fluorescence. Lane 1, $20 \mu \mathrm{g}$ nanodiscs alone; lane 2, $1 \mu \mathrm{g}$ Bax plus nanodiscs plus $50 \mu \mathrm{M}$ Bid BH3 peptide. Lane 3, $1 \mu \mathrm{g}$ Bax protein alone. Fluorescein-maleimide-labeled Bax visualized by UV indicates that association with nanodiscs is dependent on Bid (BH3) peptides. (b) Purified recombinant Bax, FITC Bid BH3 peptide and nanodiscs were analyzed by native gel followed by Coumassie Blue staining and UV fluorescence. Lane 1, $20 \mu \mathrm{g}$ nanodiscs alone; lane 2, nanodiscs plus $50 \mu \mathrm{M}$ of FITC-Bid peptide. Lane 3, nanodiscs, $1 \mu \mathrm{g}$ Bax protein and FITC-Bid FITC-Bid visualized by UV indicates that association with nanodiscs. (c) Purified recombinant Bax and nanodisc were analyzed by native gel-electrophoresis. Proteins were transferred to nitrocellulose for detection of Bax by immunoblotting. Immunoblotting data showed that Bax integrates into the nanodisc only when Bid BH3 peptide is present. Lane 1, $1 \mu \mathrm{g} \mathrm{Bax} \mathrm{protein} \mathrm{alone;} \mathrm{lane} \mathrm{2,} 20 \mu \mathrm{g}$ nanodisc alone; lane 3, Bax plus nanodisc; lane 4, Bax plus nanodisc plus $50 \mu \mathrm{M}$ of Bid BH3 peptide; lane 5, Bax plus nanodisc plus $50 \mu \mathrm{m}$ Bid BH3 mutant (G/A). (d) Purified recombinant Bax, Bcl- $X_{L}$ and nanodiscs were analyzed by native gel-electrophoresis, then the stained gel section containing the disc were excised and soaked in sample buffer at $4{ }^{\circ} \mathrm{C}$ overnight, then boiled in SDS loading buffer, loaded on SDS-PAGE gel for detection of Bax followed by immunoblotting. Lane $1,1 \mu \mathrm{g}$ Bax protein plus $20 \mu \mathrm{g}$ nanodisc; lane 2, $1 \mu \mathrm{g}$ Bax protein plus $20 \mu \mathrm{g}$ nanodisc and $50 \mu \mathrm{M}$ of Bid BH3 peptide; lane 3, Bax plus nanodisc and $50 \mu \mathrm{g}$ of Bcl- $\mathrm{X}_{\mathrm{L}}$; lane 4, Bax plus nanodisc, $50 \mu \mathrm{M}$ of Bid $\mathrm{BH} 3$ peptide and $50 \mu \mathrm{g}$ of $\mathrm{Bcl}-\mathrm{X}_{\mathrm{L}}$. The analysis confirms that Bax only enters nanodiscs in the presence of Bid BH3 peptide and also shows that Bcl- $\mathrm{X}_{\mathrm{L}}$ abolishes Bax integration induced by Bid BH3 peptide

reconstructions of bare nanodiscs and various nanodiscBax assemblies characterized by the above-mentioned biochemical assays (Figures 2 and 3). We used cryo-EM conditions to guarantee that the samples are captured while fully hydrated and suspended in buffer, thus closely capturing their physiological environment. Carrying out the analysis in 3D allows efficient distinction between prolate and disc-like geometries of the nanodiscs. All 3D structures were generated and quantified using unbiased iterative multi-reference single-particle 3D reconstruction techniques. ${ }^{19,20}$ Sorting as well as reference-free classification protocols were used throughout to ascertain the inclusion of homogenous populations into the relevant 3D reconstruction and to test for size variations. For each reconstruction, several starting models as well as cross-validation protocols were used to ensure the absence of model bias (Supplementary Figure S2). Despite of clear indications of symmetry, all reconstructions were performed without imposing symmetry.

Bare nanodiscs were reconstructed independently from data sets collected at $\mathrm{pH} 4$ or at $\mathrm{pH}$ 7. Consistent with the previous biophysical analyses, ${ }^{14}$ we did not find discernible variations in size or shape of the bare nanodisc samples (Figures $2 b$ and 3b, Supplementary Figure S1). No significant difference was observed in the 3D structures of nanodiscs obtained at either $\mathrm{pH} 4$ or $\mathrm{pH} 7$ (Supplementary Figure S1). In the $3 \mathrm{D}$ reconstructions, bare nanodiscs adopt an elliptical configuration measuring $15 \times 11 \times 5.5 \mathrm{~nm}$ (Figure 2a). This shape is compatible with an elliptical-shaped lipid bilayer delimited by a protein belt (Figure 3a), and is similar to the shape and size derived by small angle X-ray scattering or neutron scattering. ${ }^{21}$ The shape is not compatible the previously proposed super-helical prolate configuration ${ }^{22}$ or with the circular $2 \mathrm{D}$ projection images seen in negative stain electron microscopy, ${ }^{23}$ where samples are absorbed on carbon films, subjected to fixation with high heavy-metal salt concentrations, and air dried.

Bax-monomer integration in the presence of Bid $\mathrm{BH} 3$ peptide generates $\sim 3.5-\mathrm{nm}$ diameter pores in nanodisc membranes. In addition to the analysis of bare nanodiscs, image processing was performed independently for nanodiscs with Bid $\mathrm{BH} 3$ peptide (Figure $3 \mathrm{c}$ ), and with $\mathrm{Bid} \mathrm{BH} 3$ peptide and full-length $\mathrm{Bax}(\mathrm{pH} 4$ and 7, Figures $2 \mathrm{~b}$ and $3 \mathrm{~d}$, Supplementary Figure S1). All experiments were conducted at substoichiometric concentrations of Bax and excess of nanodiscs to further disfavor the formation of oligomeric Bax species in the nanodiscs. For each condition, two to three independent biochemical preparations were used to minimize experimental bias. In total, $>11000$ images of individual particles, selected from 287 micrographs, were used for the analysis (Supplementary Figure S2). 

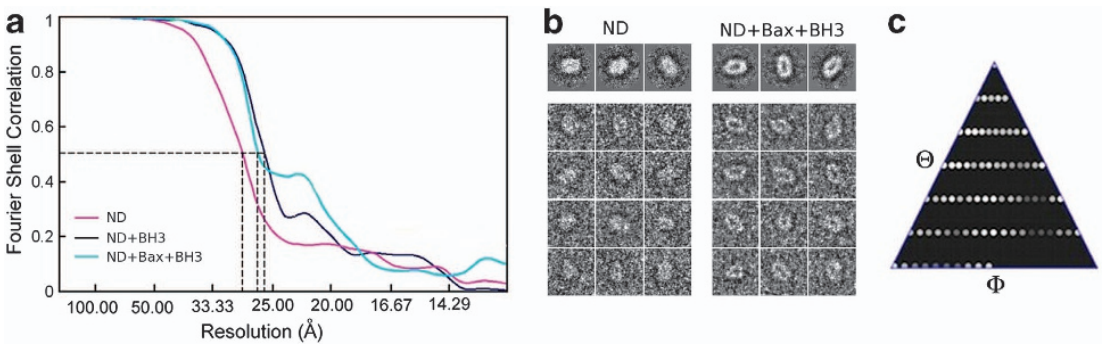

Figure 2 Cryo-EM and image processing of nanodisc assemblies. (a) The resolution of the $3 \mathrm{D}$ reconstructions is between 25 and $30 \AA$ according to the 0.5 cutoff criterion of the Fourier shell correlation (dashed lines). (b) Electron micrographs of fully hydrated bare nanodiscs (left) or nanodiscs in the presence of both Bid BH3 peptide and Bax (predominant population after sorting, right), at pH 7. The top row shows class averages after 2D reference-free classification; the other rows show representative raw images. Only views that are close to perpendicular to the membrane are shown. No fixation or dehydration steps were used during sample preparation and cryo-EM data collection. Thus, the density presented is directly proportional to the charge density of the proteins and lipids. The oval shape of the nanodiscs is discernible in many views. Holes can be clearly seen in the images of nanodiscs in the presence of Bid BH3 peptide and Bax, indicating pore formation. (c) Coverage of projection space for the bare nanodisc data. Each dot represents an orientation (Euler angles $\Theta, \Phi$ ) with the brightness proportional to the contributing number of images. The diagram shows that the reconstruction does not suffer significantly from missing views. The coverage for the other data sets is similar to that shown here
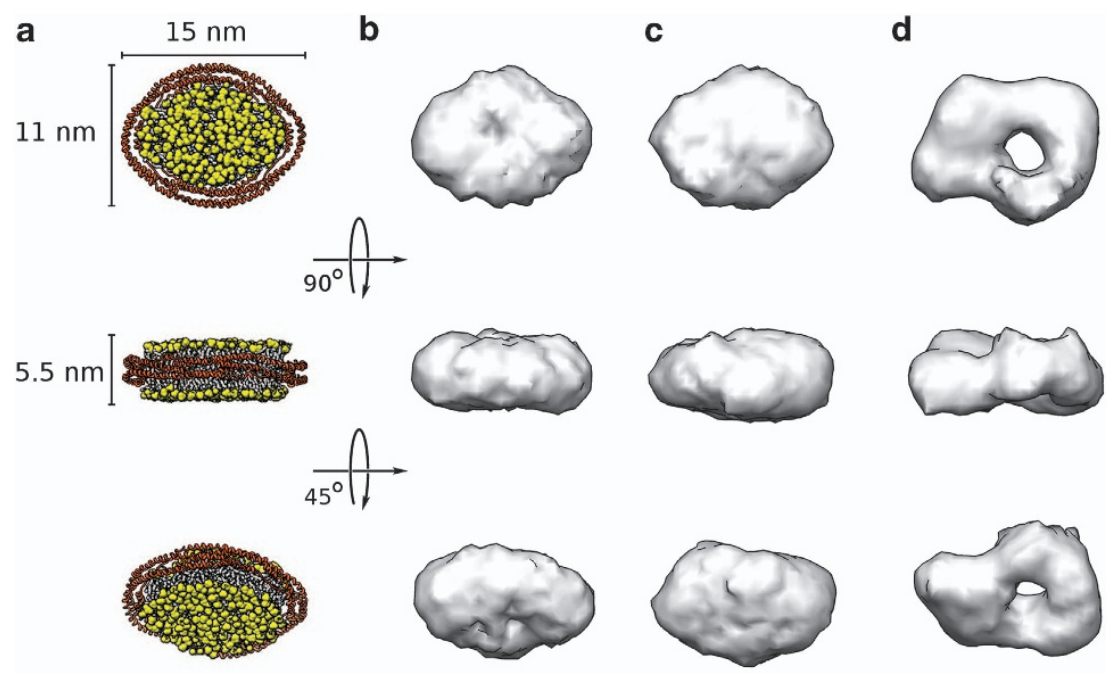

Model

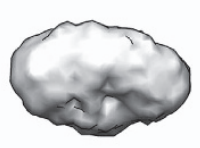

Nanodisc (ND)

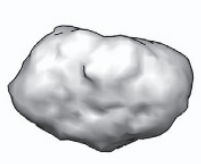

$\mathrm{ND}+\mathrm{BH} 3$

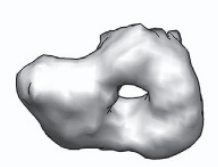

$\mathrm{ND}+\mathrm{Bax}+\mathrm{BH} 3$

Figure 3 Three-dimensional reconstructions of nanodisc assemblies. (a) Model of nanodisc derived from the 3D density. The stabilizing protein belt is shown in orange cartoon representation, the lipid bilayer is shown in yellow and white. (b) The 3D reconstruction of nanodisc is shown as a gray surface representation. Contour level chosen to accommodate the length of the protein belt in the circumference of the density. (c) Nanodisc with Bid BH3 peptide. This reconstruction is very similar to that of the nanodisc in (b). Contour level chosen to accommodate the length of the protein belt in the circumference of the density. (d) Nanodisc with Bax and Bid BH3 peptide (predominant sub-population). The contour level was chosen to accommodate the length of the protein belt in the circumference of the density

The reconstructions of nanodiscs in the presence of Bid $\mathrm{BH} 3$ peptide are very similar to bare nanodiscs, indicating that the presence of the peptides does not alter the structure of the membrane bilayer (Figures $3 \mathrm{~b}$ and c). As for bare nanodiscs, the sorting and reference-free alignment protocols did not indicate the presence of sub-populations for nanodiscs in the presence of $\mathrm{Bid} \mathrm{BH} 3$ peptides, which is consistent with the existence of a single band in the native gels for these experimental conditions.

In contrast, the nanodisc samples containing Bid $\mathrm{BH} 3$ peptide and Bax monomers segregated readily into two sub-populations. The existence of two structural conformers for these samples correlates with the existence of the two bands in the native gels (Figure 1), in which only one of the nanodisc bands colocalized with the Bax fluorescence or immunoblotting band but both bands contain fluorescent Bid peptide. Approximately, one-third (37\%) of the processed particles converged into a reconstruction virtually identical to those of bare nanodiscs or nanodiscs in the presence of Bid BH3 peptide (Supplementary Figure S2). However, the more prominent conformer $(63 \%)$ shows a clear hole of $\sim 3.5-\mathrm{nm}$ diameter in the fully hydrated phospholipid bilayer (Figures $2 b$, Supplementary Figure S1) and a significant distortion of the bilayer (Figure $4 b$ ). The error margin for the hole size, as determined by comparing independently derived reconstructions, is $\sim 0.3 \mathrm{~nm}$. These $3.5-\mathrm{nm}$ diameter Bax-mediated pores are large enough to allow release of ions and of small proteins such as cytochrome $\mathrm{c}$, one of the hallmarks of apoptosis. The total volume of the reconstruction, which gives an estimate of the complete phospholoipid and protein content of the reconstructed structure, reconfirms the single fluorescence band gel observation that only Bax 

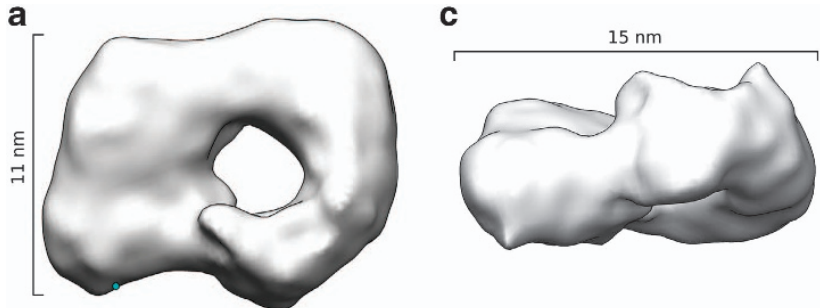

b

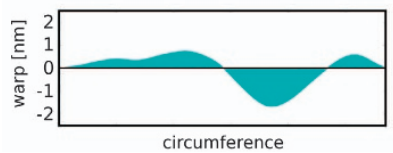

d

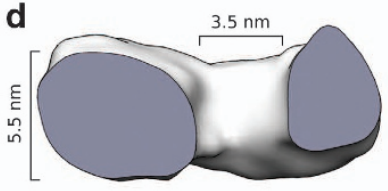

Figure 4 Bax in the presence of Bid BH3 peptide mediates membrane pore formation. (a) Top view of nanodisc with bound Bax and Bid BH3 peptide. The oval shape of the bare nanodisc (Figure $3 b$ ) is distorted and the reconstruction clearly shows a $\sim 3.5-\mathrm{nm}$ hole indicating pore formation. (b) Deviation from flat geometry around the circumference of nanodisc with bound Bax and Bid BH3 peptide showing a substantial distortion of the disc geometry. (c) Side view of nanodisc with bound Bax and Bid BH3 peptide. (d) Side view of nanodisc with bound Bax and Bid BH3 peptide with front part cut away showing the pore in transverse. The thickness at the rim of the pore is consistent with the size of the lipid bilayer

monomers can be present while higher-order Bax oligomers cannot be accommodated.

Interestingly, our reconstructions contained no discernible compact extra globular density that would account for the folded Bax-monomer solution structure, ${ }^{12}$ which is in stark contrast with other recent nanodisc studies where integrated proteins such as integrin receptors, anthrax toxin or soluble NSF attachment protein receptor (SNARE) proteins are clearly visible. ${ }^{23-25}$ This observation suggests that $\mathrm{Bax}$ is unfolded on top of the nanodisc, with its amphiphilic portions inserted into the nanodiscs in a similar way to what was proposed for colicin. ${ }^{26}$ Such unfolding of Bax is consistent with the finding that a substantial structural reorganization of Bax occurs during $\mathrm{BH} 3$-triggered activation. ${ }^{27}$

\section{Discussion}

Here, we present the first 3D structures of Bax membrane assemblies. We used biochemical, cell-free assays, cryoelectron microscopy and image processing to characterize fulllength $\mathrm{Bax}, \mathrm{Bid} \mathrm{BH} 3$ peptides and $\mathrm{Bcl}-\mathrm{xL}$ incorporation into nanometer-scale, protein-supported phospholipid bilayer discs. ${ }^{14}$ These nanodiscs have been shown to provide the means to study integral membrane proteins such as receptors, transporters and enzymes in a native-like bilayer environment. ${ }^{28}$ The ability of this bilayer system to maintain the functional activity of proteins was also shown by recent studies investigating, for example, the binding of ligands to the extracellular and cytoplasmic faces of cell membrane receptors. ${ }^{23} \mathrm{We}$ harnessed the nanodisc system and cryo-EM to derive molecular-resolution details of fully hydrated membrane phospholipid systems in the presence of Bax monomers activated by Bid BH3 peptides. Our studies showed that, as captured by native gels and direct imaging, monomeric Bax in the presence of Bid $\mathrm{BH} 3$ peptide induces the formation of 3.5-nm pores in the membrane. Our studies provide the molecular basis for the recently reported kinetic uncoupling of Bax oligomerization and pore formation in mitochondrial outer membrane vesicles. ${ }^{29}$ The structural insights described here provide evidence that BH3-peptide activation and membrane insertion of monomeric Bax are sufficient, at least in principle, to allow the initial release of ions and proteinaceous content from the outer mitrochondrial membrane, suggesting that Bax monomers are key functional units associated with the onset of Bax-mediated MOMP.

In our system, Bid $\mathrm{BH} 3$ peptide alone was found associated with nanodiscs while Bax alone was not. However, in the presence of $\mathrm{Bid} \mathrm{BH} 3$ peptide, Bax readily integrated into the lipid bilayer and caused the formation of a pore. In the cell or in cell-free assays with cellular extracts, Bax can reversibly bind to the mitochondrial membranes without fully integrating or pore formation. ${ }^{5,30}$ As full-length Bax does not associate with nanodiscs by itself, these findings indicate that other regulatory elements contribute to the control of membrane association of Bax monomers. Indeed, the composition of the phospholipid bilayer was shown previously to have profound effects on the kinetics and functionality of Bax-mediated pore formation. ${ }^{5,31,32}$ Other regulatory proteins and trafficking mechanisms may also be involved in facilitating Bax activation. ${ }^{12,33}$

The limited number of Bax helices that can be inserted in the nanodisc membrane (a maximum of four) cannot support a 3.5-nm pore that is fully lined by protein in the traditional transmembrane helix configuration. Thus, the latter indicates that the observed Bax-monomer-mediated pores are of mixed protein-lipidic nature, consistent with the fact that some Bax-derived peptides can also mediate the formation of protein-lipidic pores. ${ }^{34-37}$

The data presented here does not contain information about the actual flux of material through the initial, Bax-monomermediated pore. As the size of this initial pore and the size of cytochrome $\mathrm{C}$ are approximately the same and the charge of the lipidic head groups lining the pore may interfere with protein release, it is possible that the postulated flux of cytochrome $\mathrm{C}$ through this pore could be rather slow, with only occasional molecules finding their way through the membrane. However, once larger amounts of membrane-integrated Bax become concentrated in a membrane region, oligomerization would trigger an increase in membrane permeability by local membrane destabilization, leading to large protein-lipidic pores as observed previously by cryo-EM of large unilamellar vesicles. ${ }^{13}$ An initially slow flux would be consistent with the observation that permeabilization initially appears to be significantly slower than either Bax integration or oligomerization. ${ }^{6}$ A slow flux through the monomer pore would also explain why measurements based on continuous release kinetics from liposomes generally indicate that pore formation requires oligomerization. ${ }^{38,39}$ The measurements would be dominated by the oligomerization-triggered fast release mode and the initial slow release mode would be masked.

The formation of Bax-monomer-induced lipidic pores in the initial stage of MOMP is attractive for several reasons. First, the minimal protein requirement favors faster induction of the pro-death signaling cascade, similar to the efficient process implemented by bacterial toxins. Second, the model explains catastrophic propagation. Once self-association and oligomerization proceed, the joining of lipidic pores can be achieved with far less conformational adaptation than pores requiring adjoining protein-lined pores. The latter has been associated 
with the formation of gate-selective pore size, which also maintains cell survival, whereas the lipidic pores in the case of Bax or toxins were observed to adopt a large, inhomogeneous range of pore sizes, $25-100 \mathrm{~nm}$, which promote cell death. Third, the model also justifies the prominent role of $\mathrm{Bcl}-\mathrm{xL}$ in dysregulating apoptosis by preventing Bax insertion into the membrane. Our studies provide a new set of tools and previously not accessible data to gain new understanding of the molecular basis of MOMP, suggesting that the minimal requirement for initiation of MOMP requires Bax, a phospholipid bilayer and Bid $\mathrm{BH} 3$ peptide. Subsequent oligomerization could provide the driving force for Bax-mediated cytochrome $\mathrm{C}$ release while the lipidic nature of the pores is maintained. ${ }^{13}$

\begin{abstract}
Materials and Methods
Bax, Bcl-xL, tBid and lipid nanodiscs. Recombinant human full-length Bax, tBid and Bcl-xL with transmembrane domain removed (Bcl-xL $\Delta T M)-(\Delta T M$, lacking the c-terminal transmembrane domain) proteins were expressed and purified as $\mathrm{N}$-terminal His-tagged fusion proteins in $E$. coli and their biochemical activities were characterized as was previously described. ${ }^{12,15,40}$ For fluorescence experiments, Bax was subsequently incubated with two molar excess of fluorescein-maleimide in $20 \mathrm{mM} \mathrm{HEPES}, 50 \mathrm{mM} \mathrm{NaCl}, \mathrm{pH} 8.0$, overnight at $4{ }^{\circ} \mathrm{C}$ (Molecular Probes Kit \#F6433, Invitrogen, Life Technologies, Grand Island, NY, USA). Bid peptides (\#2036A - EDIIRNIARHLAQVGDSMDR) or fluorescein isothiocyanate (FITC) labeled Bid BH3 peptide (\#2076B - FITCAhx; \#2036A) and control $^{8}$ (\#2102 - EDIIRNIARHLAQVADSMDR) were provided by Dr. Satterthwait (Sanford-Burnham Medical Research Institute, La Jolla, CA, USA). Phospholipid membrane scaffold protein 1 (MSP1)/dipalmitoylphosphatidylcholine
\end{abstract} (DPPC) nanodiscs were generated as previously described. ${ }^{14}$

Incorporation of Bax into nanodiscs. Proteins and nanodiscs were mixed to the following final reaction concentrations: $1 \mu \mathrm{g}$ Bax, $20 \mu \mathrm{g}$ MSP1/DPPC nanodiscs, $20 \mu \mathrm{M}$ Bid peptide (or control peptide) and $50 \mu \mathrm{g} \mathrm{Bcl-xL,} 70 \mathrm{mM}$ citratephosphate buffers ( $\mathrm{pH} 4.0-8.0)$. Reactions were incubated at room temperature for $30 \mathrm{~min}$ and analyzed on 4-20\% Tris-glycine native PAGE gels (Invitrogen), or the entire reaction mixture was directly processed for cryo-EM.

Western blotting and native PAGE. Blue native gel electrophoresis was performed using the native PAGE system by Invitrogen, according to the manufacturer's instructions. Briefly, purified recombinant Bax $(1 \mu \mathrm{g})$, Bcl-xL $(50 \mu \mathrm{g})$, Bid BH3 peptides $(50 \mu \mathrm{M})$ and nanodiscs $(20 \mu \mathrm{g})$ were mixed at room temperature for $30 \mathrm{~min}$ and analyzed by $4-16 \%$ Tris-Bis native PAGE gel. The gel sections that contained nanodiscs were excised and soaked overnight, then briefly boiled in $10 \%$ SDS $(5 \mathrm{~min}$ ) before transferred to PVDF membrane. Both sample buffer and gel were loaded into denaturating gels for SDS-PAGE and followed by detection of Bax by immunoblotting using Bax antibody N2O (Santa Cruz Biotechnology, Inc., Dallas, TX, USA).

Electron cryo-microscopy. Samples from reaction mixtures described above were applied to a holey carbon film 400 mesh copper grid (Quantifoil Micro Tools GmbH, Jena, Germany) and manually plunge-frozen in liquid-nitrogencooled liquified ethane. Images of the vitrified nanodisc samples suspended over holes were obtained under low-dose conditions using a 626 single tilt liquid nitrogen cryo transfer holder (Gatan Inc., Pleasanton, CA, USA) and either a Tecnai G2 T12 Twin transmission electron microscope (FEl Company, Hillsboro, OR, USA) equipped with a Lab6 filament (Kimball Physics Inc., Wilton, NH, USA) operated at $120 \mathrm{kV}$ or a Tecnai F20 Twin transmission electron microscope (FEI Company) equipped with an FEG operated at $200 \mathrm{kV}$. Micrographs were recorded on Kodak ISO-163 plates (Eastman Kodak Co., Rochester, NY, USA) with a total dose of $20-50 \mathrm{e}^{-} / \mathrm{A}^{2}$ and under defocus ranging between 1.5 and $2.5 \mu \mathrm{m}$. The micrographs were developed in full-strength Kodak D19 developer, digitized using a SCAl scanner (Integraph, Leica Geosystems Inc., Norcross, GA, USA) at 7- $\mu \mathrm{m}$ raster and binned to a final pixel size of $0.6 \mathrm{~nm}$.

Image processing. A total of 11306 particles were interactively selected from 287 micrographs. The particles were processed and analyzed using the
EMAN2, ${ }^{41}$ SPARX ${ }^{42}$ XMIPP $^{20}$ and $\mathrm{CoAn}{ }^{43}$ software packages. Phase correction for the contrast transfer function was applied for all images using EMAN2. All data sets were subjected to reference-free classification where images were sorted according to self-similarity. This strategy does not only potentially detect subclasses caused by changes in morphology but also sub-classes caused by differences in size. The reference-free classification did not indicate sub-classes because of size differences but did indicate two sub-classes with different morphology for the data sets containing Bax and Bid BH3 peptides. Thus, each of the experimental data sets was initially sorted into two classes using models produced directly from the data using common lines. Model independence of the reconstructions obtained from the respective classes was tested by using the opposite model as a reference (Supplementary Figure S2). If model bias was detected, new references were generated from common lines of the subsets until stable, model independent reconstructions were obtained. Subdivision into two classes yielded optimal results in terms of model independence over all nanodiscBid-peptide-Bax data sets. For all other data, subdivision did not yield significantly different reconstructions than those obtained with the entire data sets. In order to obtain the highest possible quality and resolution, reference-based iterative refinement was performed using the consensus references for these conformations on the respective data subsets once model independence was confirmed. Convergence was achieved within 8-25 iterations. The resolution of the final reconstructions is between 2 and $3 \mathrm{~nm}$ according to the 0.5 Fourier shell correlation cutoff criterion (Figure 2a). The somewhat restricted resolution is consistent with the dynamic nature of nanodiscs in solution. ${ }^{44}$ The fact that the addition of particle images during the data analysis did not significantly improve the resolution at some point is also consistent with a dynamic mixture of slightly different nanodisc conformations as well as the small size of the samples by EM standards. For visualization purposes, a 2-nm low-pass filter was applied to the reconstructions. This cutoff was judged to provide the best balance between spurious high-frequency details and preservation of edge crispness at the borders of the nanodiscs.

\section{Conflict of Interest}

The authors declare no conflict of interest.

Acknowledgements. We thank Larnele Hazelwood and Chung-Yueh Ouyang for the EM sample preparation and screening, Dr. Robert Jeng for supplementary biochemical analysis, Dr. Sharon Schendel for initial biochemical preparations and Drs. Mark A McLean and Stephen G Sligar for providing the nanodisc sample. This work was supported by National Institutes of Health $(\mathrm{NIH})$ grants P01-CA069381 and R01-GM60554 (JCR). Some aspects of the work were supported by P01-GM098412 (DH) and P01-GM066311 (NV).

\section{Author contributions}

JCR, NV and DH conceived the project and wrote the paper. EK and DH designed the experiments. DZ purified and characterized the protein samples; EK characterized and optimized the sample preparation protocols. DH guided the EM experiments that were performed by MS. NV developed the image processing protocols and guided the image processing that was performed by X-PX.

1. Youle RJ, Strasser A. The BCL-2 protein family: opposing activities that mediate cell death Nat Rev Mol Cell Biol 2008; 9: 47-59.

2. Chipuk JE, Fisher JC, Dillon CP, Kriwacki RW, Kuwana T, Green DR. Mechanism of apoptosis induction by inhibition of the anti-apoptotic BCL-2 proteins. Proc Natl Acad Sci USA 2008; 105: 20327-20332.

3. Walensky LD, Gavathiotis E. BAX unleashed: the biochemical transformation of an inactive cytosolic monomer into a toxic mitochondrial pore. Trends Biochem Sci2011; 36: 642-652.

4. Martinou JC, Youle RJ. Mitochondria in apoptosis: Bcl-2 family members and mitochondrial dynamics. Dev Cell 2011; 21: 92-101.

5. Christenson E, Merlin S, Saito M, Schlesinger P. Cholesterol effects on BAX pore activation. J Mol Biol 2008; 381: 1168-1183.

6. Lovell JF, Billen LP, Bindner S, Shamas-Din A, Fradin C, Leber B et al. Membrane binding by tBid initiates an ordered series of events culminating in membrane permeabilization by Bax. Cell 2008; 135: 1074-1084.

7. Dlugosz PJ, Billen LP, Annis MG, Zhu W, Zhang Z, Lin J et al. Bcl-2 changes conformation to inhibit Bax oligomerization. EMBO J 2006; 25: 2287-2296.

8. Wang X, Silver D. Octree based algorithm for three-dimensional feature tracking. Tech Rep 1996 CAIP-TR- 20: 1-37. 
9. Oh KJ, Barbuto S, Pitter K, Morash J, Walensky LD, Korsmeyer SJ. A membrane-targeted $\mathrm{BID} B C L-2$ homology 3 peptide is sufficient for high potency activation of $\mathrm{BAX}$ in vitro. J Biol Chem 2006; 281: 36999-37008.

10. Chipuk JE, McStay GP, Bharti A, Kuwana T, Clarke CJ, Siskind LJ et al. Sphingolipid metabolism cooperates with BAK and BAX to promote the mitochondrial pathway of apoptosis. Cell 2012; 148: 988-1000.

11. Kuwana T, Bouchier-Hayes L, Chipuk JE, Bonzon C, Sullivan BA, Green DR et al. BH3 domains of $\mathrm{BH} 3$-only proteins differentially regulate Bax-mediated mitochondria membrane permeabilization both directly and indirectly. Mol Cell 2005; 17: 525-535.

12. Suzuki M, Youle RJ, Tjandra N. Structure of Bax: coregulation of dimer formation and intracellular localization. Cell 2000; 103: 645-654.

13. Schafer B, Quispe J, Choudhary V, Chipuk JE, Ajero TG, Du H et al. Mitochondrial outer membrane proteins assist Bid in Bax-mediated lipidic pore formation. Mol Biol Cell 2009; 20: 2276-2285

14. Denisov IG, Grinkova YV, Lazarides AA, Sligar SG. Directed self-assembly of monodisperse phospholipid bilayer nanodiscs with controlled size. J Am Chem Soc 2004; 126: 3477-3487.

15. Kuwana T, Mackey MR, Perkins G, Ellisman MH, Latterich M, Schneiter R et al. Bid, Bax and lipids cooperate to form supramolecular openings in the outer mitochondrial membrane. Cell 2002; 111: 331-342.

16. Wolter KG, Hsu YT, Smith CL, Nechushtan A, Xi XG, Youle RJ. Movement of Bax from the cytosol to mitochondria during apoptosis. J Cell Biol 1997; 139: 1281-1292.

17. Antonsson B, Montessuit S, Sanchez B, Martinou JC. Bax is present as a high molecular weight oligomer/complex in the mitochondrial membrane of apoptotic cells. J Biol Chem 2001; 276: 11615-11623.

18. Shaw AW, McLean MA, Sligar SG. Phospholipid phase transitions in homogeneous nanometer scale bilayer discs. FEBS Lett 2004; 556: 260-264.

19. Spahn CM, Penczek PA. Exploring conformational modes of macromolecular assemblies by multiparticle cryo-EM. Curr Opin Struct Biol 2009; 19: 623-631.

20. Scheres SH, Gao H, Valle M, Herman GT, Eggermont PP, Frank J et al. Disentangling conformational states of macromolecules in 3D-EM through likelihood optimization. Nat Methods 2007; 4: 27-29.

21. Skar-Gislinge N, Simonsen JB, Mortensen K, Feidenhans'I R, Sligar SG, Lindberg Møller B et a. Elliptical structure of phospholipid bilayer nanodiscs encapsulated by scaffold proteins: casting the roles of the lipids and the protein. J Am Chem Soc 2010; 132: 13713-13722.

22. Wu Z, Gogonea V, Lee X, Wagner MA, Li XM, Huang Y et al. Double superhelix model of high density lipoprotein. J Biol Chem 2009; 284: 36605-36619.

23. Ye F, Hu G, Taylor D, Ratnikov B, Bobkov AA, McLean MA et al. Recreation of the terminal events in physiological integrin activation. J Cell Biol 2010; 188: 157-173.

24. Katayama H, Wang J, Tama F, Chollet L, Gogol EP, Collier RJ et al. Three-dimensiona structure of the anthrax toxin pore inserted into lipid nanodiscs and lipid vesicles. Proc Nat Acad Sci USA 2010; 107: 3453-3457.

25. Shi L, Shen QT, Kiel A, Wang J, Wang HW, Melia TJ et al. SNARE proteins: one to fuse and three to keep the nascent fusion pore open. Science 2012; 335: 1355-1359.

26. Sobko AA, Kotova EA, Antonenko YN, Zakharov SD, Cramer WA. Effect of lipids with different spontaneous curvature on the channel activity of colicin E1: evidence in favor of a toroidal pore. FEBS Lett 2004; 576: 205-210.

27. Gavathiotis E, Reyna DE, Davis ML, Bird GH, Walensky LD. BH3-triggered structura reorganization drives the activation of proapoptotic BAX. Mol Cell 2010; 40: 481-492.
28. Bayburt TH, Sligar SG. Membrane protein assembly into nanodiscs. FEBS Lett 2010; 584 : $1721-1727$

29. Kushnareva Y, Andreyev AY, Kuwana T, Newmeyer DD. Bax activation initiates the assembly of a multimeric catalyst that facilitates Bax pore formation in mitochondrial outer membranes. PLoS Biol 2012; 10: e1001394.

30. Edlich F, Baneriee S, Suzuki M, Cleland MM, Arnoult D, Wang C et al. Bcl-x $(\mathrm{L})$ retrotranslocates Bax from the mitochondria into the cytosol. Cell 2011; 145: 104-116.

31. Martínez-Abundis E, Correa F, Rodríguez E, Soria-Castro E, Rodríguez-Zavala JS, Pacheco-Alvarez D et al. CRAC-like motif in BAX sequence: relationship with protein insertion and pore activity in liposomes. Biochim Biophys Acta 2011; 1808: 1888-1895.

32. Landeta O, Landajuela A, Gil D, Taneva S, Di Primo C, Sot B et al. Reconstitution of proapoptotic BAK function in liposomes reveals a dual role for mitochondrial lipids in the BAK-driven membrane permeabilization process. J Biol Chem 2011; 286: 8213-8230.

33. Guo B, Zhai D, Cabezas E, Welsh K, Nouraini S, Satterthwait AC et al. Humanin peptide suppresses apoptosis by interfering with Bax activation. Nature 2003; 423: 456-461.

34. Qian S, Wang W, Yang L, Huang HW. Structure of transmembrane pore induced by Bax-derived peptide: evidence for lipidic pores. Proc Natl Acad Sci USA 2008; 105 . 17379-17383

35. Epand RF, Martinou JC, Montessuit S, Epand RM. Transbilayer lipid diffusion promoted by Bax: implications for apoptosis. Biochemistry 2003; 42: 14576-14582.

36. Garcia-Saez AJ, Coraiola M, Serra MD, Mingarro I, Muller P, Salgado J. Peptides corresponding to helices 5 and 6 of Bax can independently form large lipid pores. FEBS J 2006; 273: 971-981.

37. Fuertes G, Garcia-Saez AJ, Esteban-Martin S, Gimenez D, Sanchez-Munoz OL, Schwille $\mathrm{P}$ et al. Pores formed by baxalpha5 relax to a smaller size and keep at equilibrium. Biophys J 2010; 99: 2917-2925.

38. Saito M, Korsmeyer SJ, Schlesinger PH. BAX-dependent transport of cytochrome c reconstituted in pure liposomes. Nat Cell Biol 2000; 2: 553-555.

39. Schlesinger PH, Saito M. The Bax pore in liposomes, biophysics. Cell Death Differ 2006; 13: $1403-1408$.

40. Zhai D, Ke N, Zhang H, Ladror U, Joseph M, Eichinger A et al. Characterization of the antiapoptotic mechanism of Bcl-B. Biochem J 2003; 376: 229-236.

41. Tang G, Peng L, Baldwin PR, Mann DS, Jiang W, Rees I et al. EMAN2: an extensible image processing suite for electron microscopy. J Struct Biol 2006; 157: 38-46.

42. Hohn M, Tang G, Goodyear G, Baldwin PR, Huang Z, Penczek PA et al. SPARX, a new environment for cryo-EM image processing. J Struct Biol 2007; 157: 47-55.

43. Volkmann N, Hanein D. Quantitative fitting of atomic models into observed densities derived by electron microscopy. J Struct Biol 1999; 125: 176-184

44. Morgan CR, Hebling CM, Rand KD, Stafford DW, Jorgenson JW, Engen JR Conformational transitions in the membrane scaffold protein of phospholipid bilayer nanodiscs. Mol Cell Proteomics 2011; 10: 010876

(c) (i) (2) Cell Death and Disease is an open-access journal published by Nature Publishing Group. This work is licensed under a Creative Commons Attribution-NonCommercialShareAlike 3.0 Unported License. To view a copy of this license, visit http://creativecommons.org/licenses/by-nc-sa/3.0/ 